\title{
Less Invasive Combined Micro- and Endoscopic Neurolysis of Superficial Peroneal Nerve Entrapment: Technical Note
}

\author{
Kyongsong KIM, ${ }^{1}$ Toyohiko IsU, ${ }^{2}$ Rinko KoKUBO, ${ }^{1}$ Daijiro MORIMOTO, ${ }^{3}$ \\ Naotaka IWAMOTO, ${ }^{3}$ and Akio MORITA ${ }^{3}$ \\ ${ }^{1}$ Department of Neurological Surgery, Chiba Hokuso Hospital, Nippon Medical School, \\ Inzai, Chiba, Japan \\ ${ }^{2}$ Department of Neurosurgery, Kushiro Rosai Hospital, Kushiro, Hokkaido, Japan \\ ${ }^{3}$ Department of Neurological Surgery, Nippon Medical School, Tokyo, Japan
}

\begin{abstract}
As superficial peroneal nerve (S-PN) entrapment neuropathy is relatively rare, it may be an elusive clinical entity. For decompression surgery addressing idiopathic S-PN entrapment, narrowarea decompression may be insufficient and long-area decompression along the S-PN from the peroneus longus muscle (PLM) to the peroneal nerve exit site may be required. To render it is less invasive, we performed S-PN neurolysis in a combined microscope/endoscope procedure. We report our surgical procedure and clinical outcomes. We microsurgically decompressed the affected S-PN under local anesthesia without a proximal tourniquet. We made a small linear skin incision at the distal portion of the S-PN, performed distal decompression of the S-PN where it penetrated the deep fascia, and then performed proximal decompression under an endoscope. At the site where the S-PN exited the PLM, we placed additional small incisions and proceeded to microscopic decompression. We surgically treated three patients with S-PN entrapment. They were two men and one woman ranging in age from 66 to 85 years. The mean postoperative follow-up was 22 months. Their symptoms before treatment and at the latest follow-up visit were recorded on the numerical rating scale (NRS). The mean incision length was $5.5 \mathrm{~cm}$ and $17.3 \mathrm{~cm}$ of the S-PN was decompressed. All three patients reported postoperative symptom improvement. There were no complications. In patients with idiopathic S-PN entrapment, long-site neurolysis under local anesthesia using a microscope/endoscope combination is useful.
\end{abstract}

Keywords: superficial peroneal nerve, neurolysis, nerve entrapment, endoscope

\section{Introduction}

The most common neuropathy in the leg is common peroneal nerve (PN) entrapment; it is seen around the peroneal head and the symptoms are numbness, pain, and motor weakness. Symptomatic entrapment of the superficial PN (S-PN) is rare; it may occur in the antero-lateral shin and the symptoms are numbness and pain in the affected area. Styf ${ }^{11}$ diagnosed S-PN entrapment in 17 of 480 (3.5\%) patients with chronic leg pain.

Received June 17, 2020; Accepted December 7, 2020

Copyright $\subseteq 2021$ by The Japan Neurosurgical Society This work is licensed under a Creative Commons AttributionNonCommercial-NoDerivatives International License.
Good outcomes after S-PN entrapment surgery have been reported. ${ }^{2-9)}$ Elsewhere $^{10)}$ we described microsurgical S-PN decompression under local anesthesia without a proximal tourniquet. For sufficient decompression, the area from the peroneus longus muscle (PLM) to the peroneal nerve exit site along the S-PN may have to be included. This requires a long skin incision. To render our surgical method less invasive, we operated by combining the use of a microscope and an endoscope.

\section{Patients and Methods}

Our study was approved by the institutional ethics committee of Chiba Hokuso Hospital. Informed consent for the procedure was obtained from all patients. 
Table 1 Patient characteristics

\begin{tabular}{lcccccccc}
\hline Case & $\begin{array}{c}\text { Age/ } \\
\text { sex }\end{array}$ & Site & $\begin{array}{c}\text { C-PN to S-PN } \\
\text { operation* }\end{array}$ & $\begin{array}{c}\text { Follow-up } \\
\text { (months) }\end{array}$ & $\begin{array}{c}\text { S-PN } \\
\text { blocks }\end{array}$ & $\begin{array}{c}\text { S-PN surgery } \\
\text { length (cm) }\end{array}$ & $\begin{array}{c}\text { Decompression } \\
\text { length (cm) }\end{array}$ & $\begin{array}{c}\text { NRS change } \\
\text { pre/postop }\end{array}$ \\
\hline 1 & $66 / \mathrm{M}$ & lt & 25 & 50 & 1 & $5(2.5+2.5)$ & 17 & $7 / 1$ \\
2 & $85 / \mathrm{M}$ & lt & 58 & 9 & 2 & $6(2.5+1.5+2.0)$ & 18 & $8 / 2$ \\
3 & $77 / \mathrm{F}$ & lt & 7 & 7 & 5 & $5.5(3.0+2.5)$ & 17 & $6 / 1$ \\
\hline
\end{tabular}

*Interval in months and "times. C-PN: common peroneal nerve, F: female, lt: left, M: male, NRS: numerical rating scale, pre: pre-operation, postop: post-operation, S-PN: superficial peroneal nerve.

Our S-PN entrapment diagnosis was primarily based on clinical symptoms. ${ }^{10)}$ All patients complained of pain and/or paresthesia of the instep and the lateral part of the shin without paresis; the symptoms were exacerbated by walking (intermittent claudication). All manifested Tinel-like signs along the S-PN. Patients who failed to improve under conservative therapy for more than 3 months were candidates for surgery.

Between January 2016 and August 2019, we surgically treated three patients with S-PN entrapment at our institution (Table 1). They were two men and one woman; their average age was 76 years (66-85 years). Two patients (cases 1 and 3) had a history of lumbar surgery for lumbar spinal canal stenosis; their postoperative lumbar MRI scans had shown sufficient decompression and no foraminal stenosis. The other patient (case 2) presented with mild lumbar spinal canal stenosis. All symptoms were unilateral and all patients had been operated for ipsilateral common PN entrapment. Thereafter, one patient (case 1) continued experiencing mild symptoms in the narrow area of the S-PN that gradually worsened. The other two reported symptom improvement; however, their symptoms reappeared 1-3 months postoperatively in the narrow area of the S-PN.

Tinel-like signs were observed at several sites along their S-PN. Their symptoms disappeared after S-PN blocks with $2 \mathrm{~mL}$ of $1 \%$ lidocaine (average 2.7 blocks, range: 1-5 blocks). We blocked the S-PN in the shallow layer under the fascia at the Tinel point at the antero-lateral part of the lower leg. Successful S-PN blockage was confirmed when the patients recovered a radiating sense along the S-PN after the injection and reported analgesia in the affected area. The mean interval between their common PN- and their S-PN surgery was 30.0 months (range: 7-58 months). Our postoperative follow lasted a mean of 22 months (range: 7-50 months).

\section{Surgical method}

Anatomically, the S-PN branches from the common PN in the PLM and runs antero-inferiorly between the peroneus longus/brevis- and the extensor digitorum longus muscle. It then penetrates the deep fascia at the shin and runs to the instep. In all cases, we decompressed from the exit site in the PLM to a site beyond its penetration of the deep fascia.

As shown in Fig. 1a, with the patient in the supine position, the leg was slightly rotated and fixed so that the S-PN faced upward. Surgery was performed with the patient under local anesthesia, no proximal tourniquet was used. We drew a line on the leg along the S-PN; it included the area of the Tinel-like sign(s). Then we made an about $25 \mathrm{~mm}$ long skin incision approximately $10 \mathrm{~cm}$ from the lateral malleolus to identify the peripheral portion of the S-PN. We exposed and cut the fascia to identify the affected S-PN (Fig. 2). Under the microscope, the S-PN was decompressed in a distal direction and then, using the endoscope (0 degree Rigid Neuroscope, Olympus Corp., Tokyo, Japan), in the proximal direction (Figs. 1b and 3). To create space, the surface trocar (Argyle TM, Trocar Catheter 28Fr, Covidien Japan Inc., Tokyo, Japan) was removed and the S-PN in the resulting open U-shaped area was decompressed.

First, the subcutaneous layer was blindly peeled off and the trocar was inserted subcutaneously with reference to the S-PN marked before surgery on the skin. Because the trocar was slowly and deeply inserted subcutaneously above the outer fascia, the nerve under the fascia was not affected. This allowed for blind trocar insertion. We then slowly and deeply inserted the endoscope in the created space and started decompression. The fascia was incised along the S-PN using the endoscope, paying attention to S-PN variations. In the area proximal to the PLM insertion site, we placed an about $20 \mathrm{~mm}$ long incision and decompressed the S-PN under a microscope (Fig. 4).

The disappearance of the Tinel-like sign(s) was confirmed. For further confirmation of sufficient decompression, we also checked for the disappearance of dynamic compression and of the patient's symptoms by intraoperative ankle movement. After surgery, the patients were allowed to walk freely without any external fixation. They were discharged the day after surgery. 


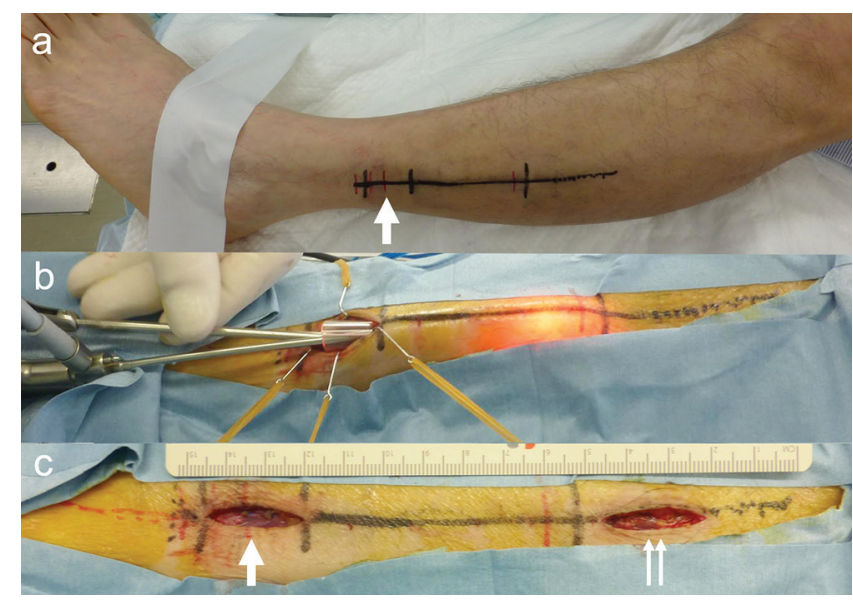

Fig. 1 (a) Marking of the planned long skin incision. We made an incision of about $25 \mathrm{~mm}$ around 1/3 on the caudal side (arrow). (b) After distal decompression of the S-PN, proximal decompression was performed with the aid of an endoscope introduced through the space made with a trocar. The endoscope and scissors are inserted into the trocar; the scissors are held in the hand. (c) After S-PN decompression. Note the distaland proximal incisions (single and double arrows, respectively). S-PN: superficial peroneal nerve.

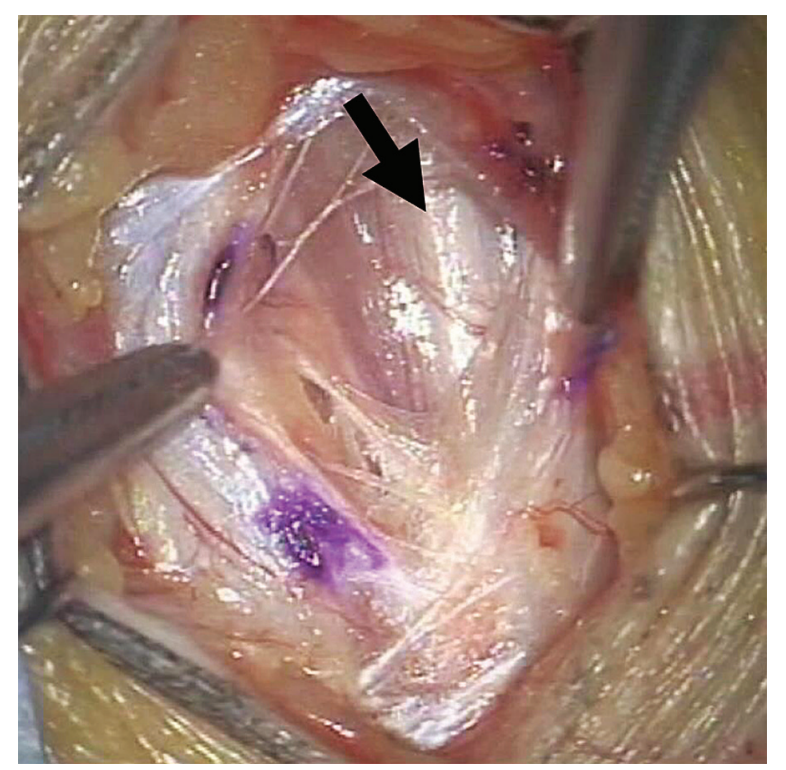

Fig. 2 Exposure of the superficial fascia. The S-PN (arrow) at the distal portion is identified after cutting the fascia. S-PN: superficial peroneal nerve.

\section{Outcome evaluation}

Using the numerical rating scale (NRS), we recorded symptom severity in the affected area before surgery and at the final follow-up visit. ${ }^{11)}$ Data were expressed as the mean \pm standard deviation.

\section{Results}

A mean S-PN length of $17.3 \mathrm{~cm}$ was decompressed using intermittent skin incisions from the PLM to the peroneal nerve exit site of the S-PN. The total length of the skin incision was a mean of $5.5 \mathrm{~cm}$ (5-6 cm) (Fig. 1c). In case 2, we placed a $15 \mathrm{~mm}$ long incision midway in the incision because connective tissue impeded trocar placement. We encountered no intraoperative complications and all Tinel-like signs disappeared.

All patients had complained of painful intermittent claudication due to S-PN entrapment. The postoperative course of all patients was good and none experienced symptom recurrence. After surgery, the NRS of the affected area improved in all patients (Table 1).

\section{Discussion}

\section{Symptoms and etiology}

$\mathrm{S}-\mathrm{PN}$ entrapment is relatively rare. As diagnosis by nerve conduction study is difficult, it is diagnosed based on symptoms, that is, numbness and pain in the affected area. ${ }^{1,3,5-10)}$ Tinel-like signs are diagnostically informative; most patients have multiple Tinel like signs along the S-PN.,10) All three of our patients exhibited these clinical characteristics. Transient symptom improvement by lidocaine-block of the S-PN is diagnostically useful,, ${ }^{4,6)}$ we also used it as a diagnostic tool for S-PN entrapment.

The etiology of S-PN entrapment is unknown. Symptoms tend to be exacerbated by walking ${ }^{1,3,5-10)}$ and S-PN entrapment may be due to dynamic compression. S-PN- and common PN entrapment may be coincident; $78 \%-100 \%$ of patients subjected to S-PN entrapment surgery had undergone common PN entrapment decompression surgery. ${ }^{8,10)}$ According to Franco et al., ${ }^{8)}$ patients with proximal site compression may be susceptible to compression at a distal site; Upton and McComas ${ }^{12)}$ referred to this as the double-crush phenomenon. These observations suggest that in patients with recurrent or persistent symptoms after common PN entrapment surgery, S-PN entrapment must be ruled out.

\section{Surgery}

S-PN entrapment may be due to muscle herniation, mass lesions, trauma, and idiopathic causes. ${ }^{1,3-9)}$ As none of our patients presented with fascial defects, trauma, or mass lesions, we made a diagnosis of idiopathic S-PN entrapment.

Most patients with idiopathic S-PN entrapment manifest Tinel-like signs at multiple sites along the S-PN. ${ }^{3,10)}$ They may experience symptom recurrence 


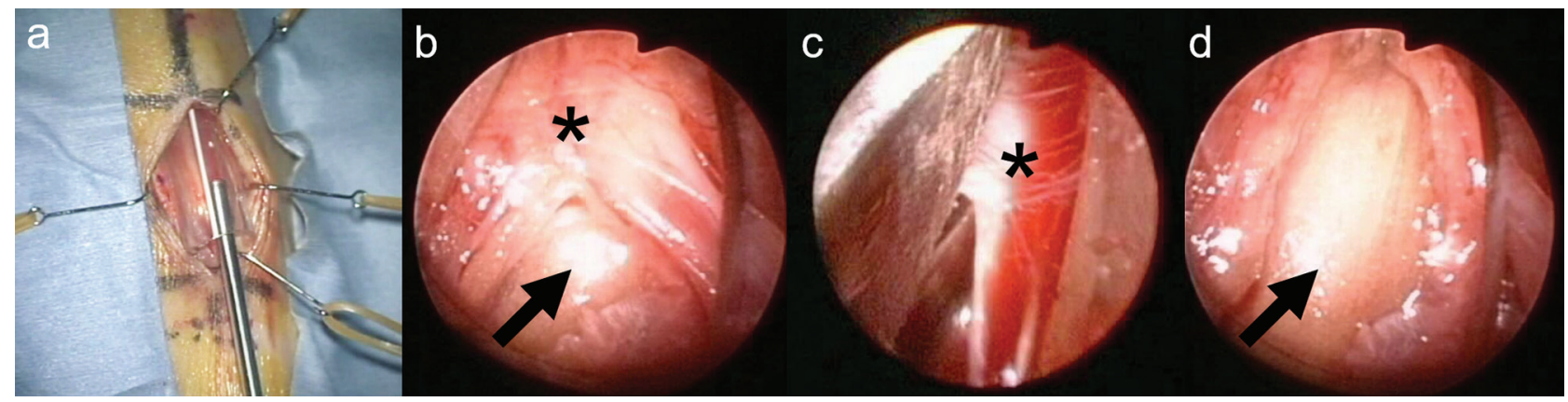

Fig. 3 Using an endoscope, the S-PN (arrow) is decompressed after cutting the fascia (*) in the proximal direction. (a) Endoscope insertion through the space made with the modified trocar. (b) Note the S-PN (arrow) under the fascia $(*)$. (c) The fascia $(*)$ is cut with the scissors. (d) Decompression of the S-PN (arrow) after cutting the fascia. S-PN: superficial peroneal nerve.

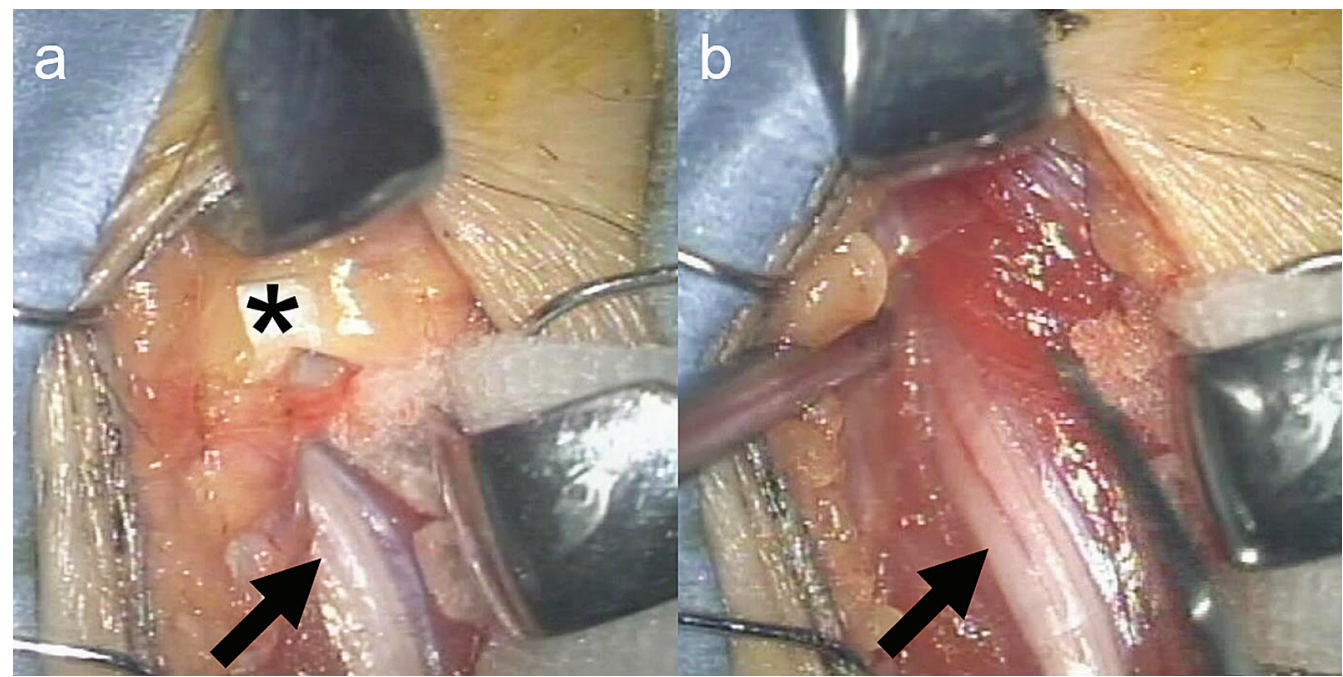

Fig. 4 In the area proximal to the site of insertion into the PLM a small, about 20 mm incision is made and the S-PN is decompressed under a microscope. (a) Note the S-PN (arrow) near its exit from the PLM. (*PLM fascia). (b) The S-PN (arrow) is completely decompressed. PLM: peroneus longus muscle, S-PN: superficial peroneal nerve.

after limited decompression surgery because postoperatively, the pressure around the S-PN is increased by leg loading. ${ }^{4)}$ Therefore, complete decompression along the anterior shin has been recommended. ${ }^{3,4,6,10)}$ Styf and Morberg ${ }^{3)}$ emphasized that the S-PN should be decompressed by complete opening to reduce the risk of irritation due to the residual fascia edge and to avoid muscle herniation. We also had encountered a patient with symptom recurrence after restricted S-PN decompression surgery. This alerts to the need for extensive decompression from the PLM to the S-PN penetration site; the procedure requires a long (mean: $13.2 \mathrm{~cm}$, range: $8-21 \mathrm{~cm}$ ) skin incision. ${ }^{10)}$

When only the proximal part of S-PN is decompressed with the aid of an endoscope only, adhesions from earlier common PN surgery are of concern.
However, common PN decompression is performed around the entrance of the PLM and the area involved in common PN surgery does not affect the S-PN decompression area. Therefore, a history of common PN surgery does not hinder proximal site decompression of S-PN with the endoscope alone. As this issue remains to be investigated, it is the subject of future studies in our institute. Our combined use of a microscope and an endoscope resulted in less invasive surgery. It may be possible to perform the procedure using only a distal incision or only an endoscope.

Wound problems are elicited by local factors such as tension applied to the wound. When the incision is long, the force on the wound edges is increased because fewer skin portions are attached. ${ }^{13)}$ As incisions longer than $10 \mathrm{~cm}$ are independently 
predictive of postoperative pain, ${ }^{14)}$ the wound length must be shortened and the procedure must be less invasive. Because the S-PN course is relatively straight after branching from the common PN, linear decompression with the aid of an endoscope is feasible. By the combined use of an endoscope and a microscope, we avoided making a linear incision in the area where decompression is relatively easy. This rendered the procedure less invasive and not cumbersome for surgeons not accustomed to using an endoscope. Because the operative space is tight, we used the trocar to increase the space, thereby contributing to the ease of surgery.

\section{Limitations}

Our study has some limitations. The patient population was small and the follow-up periods were relatively short. We will continue to follow our patients to evaluate their long-term outcomes. The clinical results of longitudinal surgical decompression techniques and our less invasive procedure need to be compared to reveal the advantages and disadvantages of our method.

\section{Conclusions}

For sufficient decompression in patients with idiopathic S-PN entrapment, extensive decompression from the PLM to the S-PN penetration site is necessary. With the patient under local anesthesia, the combined use of a microscope and an endoscope may render S-PN decompression easier and less invasive.

\section{Conflicts of Interest Disclosure}

The authors declare that they have no conflicts of interest and no commercial relationships and that they received no support from pharmaceutical or other companies. All authors who are members of The Japan Neurosurgical Society (JNS) have completed the Self-reported COI Disclosure Statement forms available at the website for JNS members.

\section{References}

1) Styf J: Entrapment of the superficial peroneal nerve. Diagnosis and treatment by decompression. J Bone Joint Surg Br 71: 131-135, 1989
2) Henry AK: Extensile Exposure. E\&S Livingstone 163, 1945

3) Styf J, Morberg P: The superficial peroneal tunnel syndrome. Results of treatment by decompression. J Bone Joint Surg Br 79: 801-803, 1997

4) Kernohan J, Levack B, Wilson JN: Entrapment of the superficial peroneal nerve. Three case reports. J Bone Joint Surg Br 67: 60-61, 1985

5) Garfin S, Mubarak SJ, Owen CA: Exertional anterolateral-compartment syndrome. Case report with fascial defect, muscle herniation, and superficial peroneal-nerve entrapment. J Bone Joint Surg Am 59: 404-405, 1977

6) Banerjee T, Koons DD: Superficial peroneal nerve entrapment. Report of two cases. J Neurosurg 55: 991-992, 1981

7) Yang LJ, Gala VC, McGillicuddy JE: Superficial peroneal nerve syndrome: An unusual nerve entrapment - case report. J Neurosurg 104: 820-823, 2006

8) Franco MJ, Phillips BZ, Lalchandani GR, Mackinnon SE: Decompression of the superficial peroneal nerve: Clinical outcomes and anatomical study. $J$ Neurosurg 126: 330-335, 2017

9) McAuliffe TB, Fiddian NJ, Browett JP: Entrapment neuropathy of the superficial peroneal nerve. A bilateral case. J Bone Joint Surg Am 67: 62-63, 1985

10) Matsumoto J, Isu T, Kim K, et al.: Clinical features and surgical treatment of superficial peroneal nerve entrapment neuropathy. Neurol Med Chir (Tokyo) 58: 320-325, 2018

11) Hjermstad MJ, Fayers PM, Haugen DF, et al.: Studies comparing numerical rating scales, verbal rating scales, and visual analogue scales for assessment of pain intensity in adults: A systematic literature review. J Pain Symptom Manage 41: 1073-1093, 2011

12) Upton AR, McComas AJ: The double crush in nerve entrapment syndromes. Lancet 2: 359-362, 1973

13) Berry JAD, Miulli DE, Lam B, et al.: The neurosurgical wound and factors that can affect cosmetic, functional, and neurological outcomes. Int Wound $J 16$ : 71-78, 2019

14) Janssen KJ, Kalkman CJ, Grobbee DE, Bonsel GJ, Moons KG, Vergouwe Y: The risk of severe postoperative pain: Modification and validation of a clinical prediction rule. Anesth Analg 107: 1330-1339, 2008

Corresponding author: Kyongsong Kim, MD, PhD

Department of Neurological Surgery, Chiba Hokuso Hospital, Nippon Medical School, 1715 Kamagari, Inzai, Chiba, Japan.

e-mail: kyongson@nms.ac.jp 